\title{
A 5-Year Prospective Study on Effect of Antimicrobial Surgical Prophylaxis in the Department of General Surgery
}

\author{
Ketan Kailash Khelwade ${ }^{1, *}$, Shrikalp Deshpande ${ }^{2}$ \\ ${ }^{1}$ Clinical Pharmacologist, CIMS Hospital, Ahmedabad, Gujarat, INDIA. \\ ${ }^{2}$ KB Institute of Pharmaceutical Education and Research, Gandhinagar, Gujarat, INDIA.
}

\begin{abstract}
Background: Antibiotic-resistant infections have elevated rates of morbidity and mortality, and antibiotic exposure is a significant trigger factor for drug resistance growth. If surgical antibiotic prophylaxis (SAP) increases risk for antibiotic-resistant infections, it may cause significant harm even if it decreases overall infection rates. The present study is planned to study the effect of antimicrobial surgical prophylaxis in the department of general surgery. Materials and Methods: This prospective study included young age people to old age people who underwent elective surgical procedures. The study was carried for 5 successive years from 2016 to 2020. Surgeries from multiple disciplines were included if SAP was considered discretionary by current guidelines. Results: According to the observations, the antibiotics were administered in all the surgical interventions and most of the pre-operatively antibiotics were administered by IV route. Graph showcases a gradual increase in antibiotic misuse both before and during the surgery. In the year 2016, the antibiotic usage in surgical prophylaxis was observed to be an average of $65.89 \%$. This value was found to be progressively increasing in the successive years. By the year of 2020 , the average hiked to the value of $97.11 \%$. This is conclusive of the decreasing misuse of antibiotics with years. Especially the recent times reaching the maximum peak, as appreciated in the graph. This could be attributed as one of the
\end{abstract}

major factors in development of resistance towards various microorganisms that has been managed. Conclusion: Surgical antibiotic prophylaxis is an important treatment technique for preventing postoperative infections, if the right antibiotics are administered at the right moment, for the right length of time, and for the right surgical procedures. It is crucial to use a narrow-spectrum antibiotic that is safe for the surgical venue. The expense of specific antibiotics and the endemicity of multi-resistant bacteria in specific units or clinics are also subject to periodic modification, so hospital surgical antibiotic prophylaxis procedures should be checked on a regular basis.

Key words: Antimicrobial stewardship, Antimicrobial resistance, Surgical antimicrobial prophylaxis, Surgical site infections, Anti-microbial Medical Audit.

Correspondence

Ketan Kailash Khelwade,

Clinical Pharmacologist, CIMS Hospital, Ahmedabad-380060, Gujarat, INDIA.

Email id: ketankhelwade@gmail.com

DOI: 10.5530/ijpi.2021.3.56

\section{INTRODUCTION}

One of the most effective public health initiatives of the last century was the invention and widespread usage of antimicrobial agents. These agents, along with increased hygiene and widespread vaccination (in countries where these are obtainable), have resulted in a significant decline in infectious mortality.

Drug production was flourishing in the earlier periods of antibiotics, but that as resistance arose, a new drug was still ready to cure the newly resistant bacteria. Between 1935 and 2003, fourteen different antibiotic types were developed. On the other hand, sudden antimicrobial growth came at a price-antimicrobial resistance. Antimicrobial tolerances are the most concerning issues in hospitals.

Antimicrobial resistance (AMR) is on the rise across the world, presenting a serious threat; this is particularly true in growing countries like India where AMR and contagious disease burdens are incredibly large. ${ }^{1}$ The overuse and abuse of Antimicrobial have long been recognised as significant contributors to the production of certain elevated numbers of resistance. ${ }^{2}$ Antibiotic use is growing globally, owing to rising wages, health care, and the burden of contagious diseases. Antibiotic intake rose by $36 \%$ in 71 nations between 2000 and 2010, with Russia, India, South Africa, China, and Brazil responsible for 3-quarters of the growth. ${ }^{3}$

The usage of antibiotics to deter surgical site infections (SSI) is known as surgical antimicrobial prophylaxis (SAP). ${ }^{4} \mathrm{SAP}$ is a successful treatment technique for minimising post-operative complications if the right antibiotics are administered at the correct amount of dose at suitable moment, and for suitable duration of time and for the right surgical procedures. ${ }^{5}$ Antimicrobials must be used effectively to avoid contamination to reduce the complications involved in surgical procedures. In hospitals, this is the most familiar explanation for antimicrobial application. ${ }^{6}$

It is linked to a high incidence of improper usage. These ineffective SAP, like improper use, administration, dose frequency and time interval, are linked to a rise in antibiotic resistance, which cause ADR and an enhanced likelihood of SSI, both of which drive an ever-increasing requirement for new medications and lead to increasing medical costs. ${ }^{7}$

Antimicrobial stewardship is described as coordinated efforts intended to encourage and maximise the proper usage of antimicrobials" ${ }^{\prime 8}$ and is a critical strategy for preserving antibiotic efficacy while also enhancing patient protection and care efficiency. The national Antimicrobial Stewardship Clinical Care Standard involves suitable SSP prescription. This norm was created for prescribers and patients in hospitals and general practises. The National Safety and Quality Health Service Standards demand that antimicrobial usage and tolerance be monitored. ${ }^{9}$ The involvement of minimum one contagious diseases-trained physician who devotes a portion of their time to the creation, execution, and operation of an antimicrobial stewardship programme is vital to its effectiveness. Further team microbiologist, ID physician, clinical pharmacist,

This is an open access article distributed under the terms of the Creative Commons Attribution-NonCommercial-ShareAlike 4.0 License, which allows others to remix, tweak, and build upon the work non-commercially, as long as the author is credited and the new creations are licensed under the identical terms. 
ICN and other were also helpful for stewardship program. Antimicrobial restriction policies, therapeutic protocols, and other steps must be monitored by an infectious diseases specialist to guarantee that they are focused on the current science and experience and would not place patients at hazard. Getting the programme headed by a contagious disease specialist can help to build reputation among the hospital's doctors and reduce the likelihood of the programme being a pharmacy-driven costcutting program. ${ }^{10}$ The present study is planned to study the effect of antimicrobial surgical prophylaxis in the department of general surgery.

\section{MATERIALS AND METHODS}

\section{Study Design}

This study was mainly focussed on surgical wards patients of CIMS (Care Institute of Medical Science) hospital. It is one of the finest hospitals in Ahmedabad with a bed size of 350. It is Gujarat's first digitised operating theatres and intensive care units (ICU) hospital. At CIMS Hospital, the first cardiac transplant was performed successfully. It performs neurospine surgery (on over 1500 patients since 2011), cardio-thoracic surgery (on over 5000 cardiac and 500 thoracic operations in the last five years), paediatric surgery, uro surgery, onco surgery, ortho surgery, and gastro surgery.

\section{Study period}

A hospital based cross-sectional analysis was performed to gather a data by reviewing the admitted patients in surgical wards of CIMS hospital. This study is based on five successive years (from 2016 to 2020). During the research era, all patients admitted to surgical wards were operated for cardiologist, orthopaedic, paediatric, gastro-intestinal, urology, neurology, and oncology surgical procedures were the source and sample community, respectively.

\section{Data Collection}

During the study, data were collected from a CIMS hospital using an organised antibiotic audit tool, that includes patient name, age, sex, surgery types, duration of drugs, and irrational use of drug, development, in surgical ward in which the patient was admitted. The prophylactic and post-operative antimicrobial drugs prescribed, frequency and route of administration, intra-operative re-dosing, and base of antimicrobial prescription for post-operative therapy. The method of procedure, type, and length of treatment, wound class, preoperative hospital stays, surgical antimicrobial prophylaxis (SAP), and complete hospital stay were all recorded.

\section{Inclusion and Exclusion criteria}

The patients undergoing surgery are included in this study. The patients undergoing medical wards are not included in this study.

Figure 1 depicts the whole procedure of the study. The steps followed in collecting the information is mentioned in it.

\section{Operational definitions}

SSI: Infections which occur within 30 days after a surgery or monitoring of surgical wound infection initiation within 90 days where an implant is inserted are referred to as surgical site infections.

SSI has been documented that after CABG, orthology, neurology, gynaecology, urology, and gastro surgery involving clean and cleancontaminated procedures without reconstruction. ${ }^{11,12}$ However, SSI has only been documented following a broad resection with a high SSI rate and rebuilding. The primary reasons that contribute to high SSI rates, as well as the most effective methods for dealing with this problem, remain unknown. The most significant risk factors for SSI infection after surgery,
Patients admitted in CIMS hospital

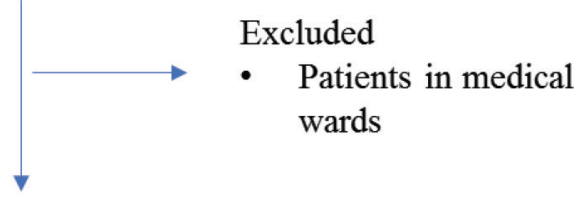

Enrolled $(\mathrm{n}=320)$

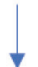

Allocated $(n=320)$

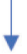

Given surgical prophylaxis $(n=320)$

\section{Measurement and analysis were taken for 5 successive years}

Figure 1: Consort flow diagram.

as well as effective prevention measures. Patients with postoperative SSI who had had CABG, orthology, neurology, gynaecology, urology, or gastro reconstructive operations were statistically analysed.

\section{SSI Strategy and Diagnostic Method}

The principal strategies for preventing SSI after CABG, orthology, neurology, gynaecology, urology, gastro reconstructive surgery at CIMS hospital were: (1) Cefmetazole is given to all patients as a preventive antibacterial therapy 30-60 min before to incision and for 3 days following surgery. (2) Povidone iodine is used to sterilise the surgical field. (3) The location is cleaned with more than $1 \mathrm{~L}$ of saline after the oropharyngeal and cutaneous areas have been divided with a flap. (4) A close-suction drainage procedure is used. (5) A mandibular reconstruction plate or an artificial tool is utilised after the surgical area has been cleaned with saline.

A sample is taken from the possibly contaminated field and cultured for bacteria if an SSI is detected. As per the canters for Disease Control and Prevention, a tiny leakage which does not come into contact with the skin is not considered an SSI. A fistula, characterised as orocutaneous or pharyngocutaneous leaking, is regarded an SSI regardless of the source.

\section{SAP}

It is the usage of antibiotics to avoid SSIs during surgery. It does not require pre-operative decolonization or care of existing infections. In CIMS hospitals, it is the most frequent reason for antimicrobial usage. Inappropriate usage, like prolonged surgical prophylaxis (e.g., five days of cefalexin after discharge), adds to the community's total burden of antibiotic use and exposing patients to adverse effects and Clostridium difficile infections. Over the years from 2016 to 2019, the proper use of antibiotics during or after surgery rose.

\section{RESULTS}

For the present prospective study, the inclusion of all types of patients to accommodate different age ranged subjects was aimed. From the young age people to the old-aged people indicated for or undergoing surgeries were included for the present trial. The age of participants observed were in the range of 5 to 87 years. 
Table 1: Patient demographics.

\begin{tabular}{|c|c|c|c|}
\hline $\begin{array}{l}\text { Variable Presented as } \\
\text { n (\%) or median (IQR) }\end{array}$ & $\begin{array}{l}\text { Pre-Shortage } \\
\text { group }(n=103)\end{array}$ & $\begin{array}{c}\text { Shortage } \\
\text { group }(n=217)\end{array}$ & $P$-Value \\
\hline Age, years & $57(38-67)$ & $58(44-70)$ & 0.19 \\
\hline Gender, male & $58(56.3)$ & $124(57.1)$ & 0.36 \\
\hline \multicolumn{4}{|c|}{ Co-morbid conditions } \\
\hline 1. Diabetes & $43(41.7)$ & $69(31.8)$ & 0.07 \\
\hline 2. Dyslipidemia & $21(20.4)$ & $42(19.4)$ & 0.80 \\
\hline 3. Hypertension & $63(61.2)$ & $133(61.3)$ & 0.98 \\
\hline 4. ESRD & $14(13.6)$ & $34(16.9)$ & 0.65 \\
\hline 5. HIV & $1(1.0)$ & $10(4.0)$ & 0.18 \\
\hline 6. Cancer & $27(26.2)$ & $43(19.8)$ & 0.18 \\
\hline 7. Transplant & $6(5.8)$ & $15(7.5)$ & 0.73 \\
\hline
\end{tabular}

Table 2: Surgical prophylaxis parametric analysis 2016-2020.

\begin{tabular}{|c|c|c|c|c|c|}
\hline Parameters & 2016 & 2017 & 2018 & 2019 & 2020 \\
\hline $\begin{array}{l}\text { Time gap not } \\
\text { maintained }\end{array}$ & $12.91 \pm 6.59$ & $4.30 \pm 2.94$ & $2.33 \pm 1.50$ & $1.88 \pm 1.64$ & $2.01 \pm 0.35$ \\
\hline $\begin{array}{c}\text { Wrong } \\
\text { documentation }\end{array}$ & $8.43 \pm 6.39$ & $4.78 \pm 2.43$ & $1.60 \pm 0.40$ & $1.00 \pm 0.70$ & $1.23 \pm 0.37$ \\
\hline $\begin{array}{c}\text { No } \\
\text { documented } \\
\text { time }\end{array}$ & $14.63 \pm 9.680$ & $3.25 \pm 1.60$ & $3.67 \pm 2.34$ & $2.0 \pm 0.94$ & $3.00 \pm 1.9$ \\
\hline $\begin{array}{c}\text { Blank } \\
\text { document }\end{array}$ & $8.33 \pm 5.17$ & $3.30 \pm 1.49$ & $3.43 \pm 1.27$ & $1.50 \pm 1.0$ & $3.11 \pm 1.75$ \\
\hline $\begin{array}{l}\text { Irrational } \\
\text { antibiotic }\end{array}$ & $5.0 \pm 4.11$ & $1.0 \pm 0.00$ & $1.00 \pm 0.00$ & $1.00 \pm 0.00$ & $1.00 \pm 0.00$ \\
\hline Overall & $39.08 \pm 21.39$ & $13.75 \pm 5.19$ & $7.33 \pm 3.77$ & $3.92 \pm 2.39$ & $5.21 \pm 2.51$ \\
\hline $\begin{array}{c}\text { Antibiotic } \\
\text { surgical } \\
\text { prophylaxis }\end{array}$ & $65.89 \pm 17.4$ & $90.02 \pm 0.20$ & $97.62 \pm 1.12$ & $98.71 \pm 0.78$ & $97.11 \pm 0.39$ \\
\hline
\end{tabular}

Table 1 displays the demographic data which includes age, sex, co-morbid conditions in both pre-shortage and shortage group. Among them the co-morbid conditions, hypertension problem was most common in both the patient group and HIV was least under the surgical prophylaxis.

Patients undergoing surgeries were given prophylactics and the data of following parameters as discussed in Table 2 were recorded. Which included the time interval, in correction or no documentation if any irrational use or misuse of antibiotics. The observation, as seen in Table 1, proved that application of stewardship program in terms of surgical prophylaxis has improved both the knowledge and outcome of the procedures progressively from 2016 to 2020 .

The application of Pearson correlation test among the observational year from 2016 to 2020 have shown promising results. Table 3 notably describes the comparison observed among these years. Statistically significant result from 2016 to 2018 was found. However, not much of the significance was observed between the years 2018-19 and 2019-20.

Graph 1 highlights the data evaluation of the antibiotic surgical prophylaxis observed in CIMS hospital. The five years of data from 2016 to 2020 are represented graphically. According to the observations, the antibiotics were administered in all the surgical interventions and most of the pre-operatively antibiotics were administered by IV route. Graph showcases a gradual increase in the rational use of antibiotic both before and during the surgery. In the year 2016, the antibiotic usage in surgical prophylaxis
Table 3: Correlation of outcome from 2016 to 2020.

\begin{tabular}{ccccc}
\hline \multicolumn{2}{c}{ Year } & Mean \pm SD & Pearson correlation & p value \\
\hline \multirow{4}{*}{2016} & 2017 & $-24.12 \pm 19.7$ & -.424 & .001 \\
& 2018 & $-31.73 \pm 17.2$ & .240 & .000 \\
& 2019 & $-32.81 \pm 17.71$ & -.330 & .000 \\
& 2020 & $-30.11 \pm 16.69$ & -.301 & .000 \\
& 2018 & $-7.60 \pm 5.04$ & -.513 & .000 \\
2017 & 2019 & $-8.69 \pm 3.95$ & .607 & .000 \\
& 2020 & $-8.01 \pm 4.11$ & .591 & .000 \\
& 2019 & $-1.08 \pm 1.65$ & -.474 & .043 \\
& 2020 & $-.97 \pm 2.21$ & -.423 & .031 \\
\hline
\end{tabular}

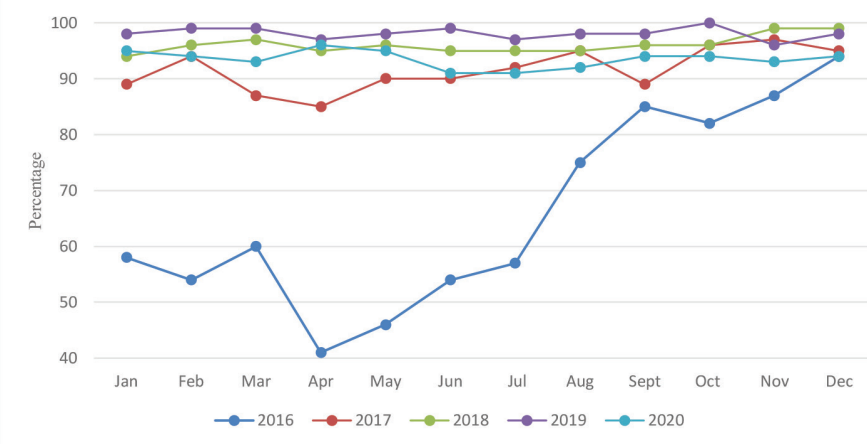

Graph 1: Antibiotic Surgical Prophylaxis 2016-2020.

was observed to be an average of $65.89 \%$. This value was found to be progressively increasing in the successive years. By the year of 2019, the average hiked to the value of $98.71 \%$. However, 2020 observed increased in the value. The increase can be attributed to COVID-19 scenario overall where the health facilities were severely challenged. Overall, this is conclusive of the increasing rational use of antibiotics with years. Especially the recent times reaching the maximum peak, as appreciated in the graph. This could be attributed as one of the major factors in development of resistance towards various microorganisms, which, by the application of stewardship program in the form of surgical prophylaxis can be easily curbed.

Among the most frequently observed surgeries at CIMS Hospital, Urology surgeries (Graph 2) pediatric cardio-thoracic surgery (Graph 3) and cardio-thoracic surgery (Graph 4 ) are few of the surgeries where surgical prophylaxis was implemented and observed.

\section{DISCUSSION}

Surgical antibiotic prophylaxis (SAP) is a short course of antibiotics provided just before operating operations to prevent post-operative surgical site infections (SSIs). SSI is attributed one of the most common healthcare associated infections (HAIs) and one of the most serious consequences of surgical procedures. Antibiotics, on the other hand, are unlikely to avoid SSI on their own, and several pre-, intra-, and postoperative requirements must be followed. ${ }^{13}$

In the present study, we have focussed on the use of antibiotics preoperatively to prevent the surgical site infections (SSI). Parenteral or intravenous mode is the appropriate choice of administration for SAP. ${ }^{14}$ In this study, most of the antibiotics was administered by IV route 


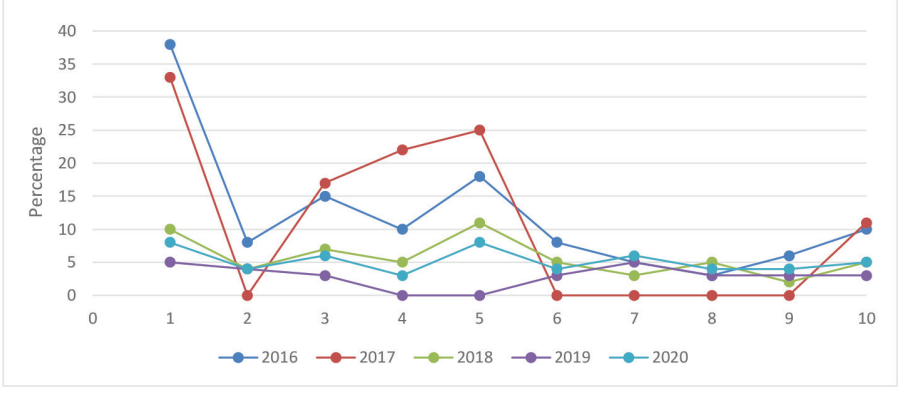

Graph 2: Urology Surgeries.

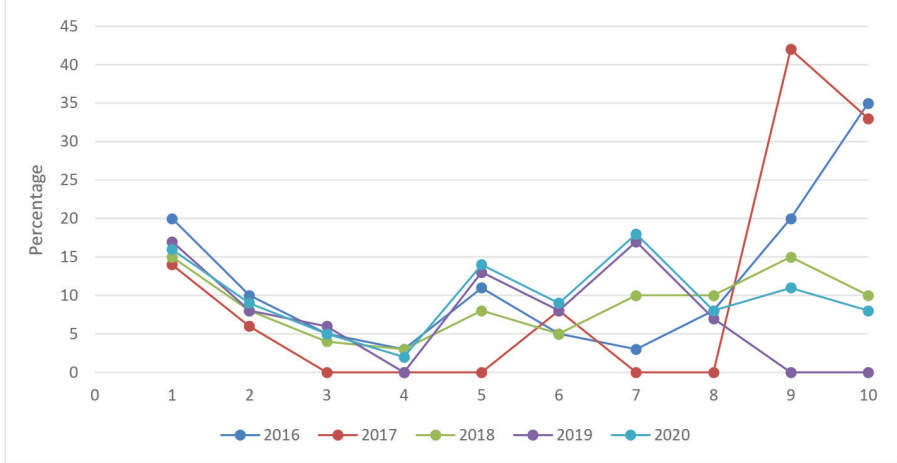

Graph 3: Pediatric Cardiothoracic.

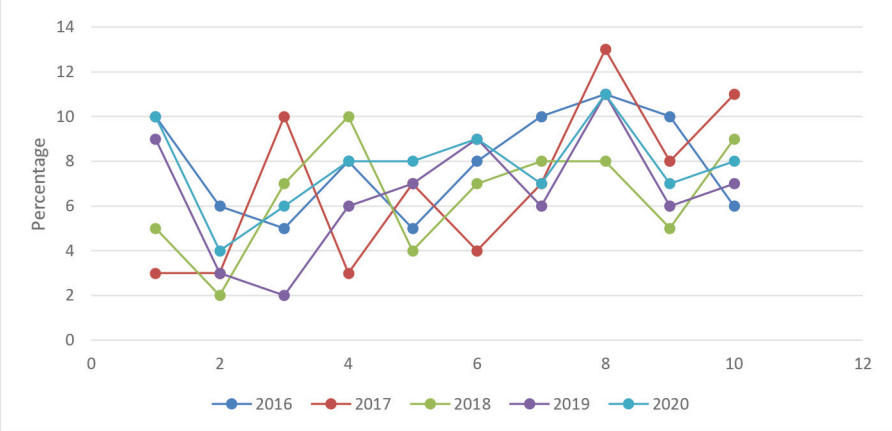

Graph 4: Cardiothoracic Surgeries.

(93.2\%), which is consistent with evidence. The result obtained in the study is in accordance with the findings of the national survey in Brisbane. ${ }^{15}$ The antibiotic over use is a legit problem in terms of development of microbial resistance. Literature supports the adverse events that follow after irrational use of antibiotics. In a study conducted by Tamma PD et al. (2017), ${ }^{16}$ increased risk was found to be associated with more antibiotic usage. In this term, the prophylactic and rationalised stewardship ensures the effective bypassing of these problems. And as the observation of the study goes, the surgical prophylaxis has improved the overall surgical outcome from the year 2016 to 2020.

This use as lead to antimicrobial resistance which is a major concern. All the patients undergoing surgery were audited and the pattern of use of antibiotics were recorded. The data collected was statistically analysed. The result obtained showed overuse of antibiotics in 2016 when compared with the result obtained from the year 2020. This sudden drop in the use of antibiotics within 5 years has led to decrease in antimicrobial resistance and decrease in efficiency of antibiotics.

The knowledge of appropriate dose is required to understand the tissueserum concentration. The breach in the balance results in toxicity which should be maintained. ${ }^{17}$ In the present study, the maintenance of time gap was documented to understand the efficacy of surgical prophylaxis. The WHO guidelines also support the dose duration, gap maintenance and choosing the right drug for surgical prophylaxis. ${ }^{18}$

The most prominent errors, according to other authors, were the timing of the first dose and the length of the prophylaxis. A retrospective research on gastro-intestinal surgery at an Israeli children's hospital found that in $32 \%$ of cases, ${ }^{14}$ the timing was right. Overuse of SAP, particularly for longer periods of time than prescribed, increases antibiotic intake, alters person and institutional microbial flora, and puts patients at risk for Clostridium difficile infection. ${ }^{19}$

\section{CONCLUSION}

Antibiotic resistance is becoming more of a problem, whether owing to inherently resistant bacteria or strains with evolved resistance. Antimicrobial selections that are more effective and cost-effective enhance results and save expenses. All the patients undergoing surgery were audited and the pattern of use of antibiotics were recorded. The result showed overuse of antibiotics in 2016 but a subsequent decrease upto year 2020. This sudden drop in the use of antibiotics within 5 years has led to decrease in antimicrobial resistance and decrease in efficiency of antibiotics. The findings of the current study support the evidence and efficiacy of surgical prophylaxis. However, more extensive studies are required to validate conclusive inferences for appropriate clinical application in evidence based protocol way.

\section{ACKNOWLEDGEMENT}

I want to thank to Department of Microbiology, CIMS Hospital for allowing doing intervention and collection of data and performing the study.

\section{ABBREVIATIONS}

SAP: Surgical antibiotic prophylaxis; AMR: Antimicrobial resistance; SSI: surgical site infections; ICN: Infection control Nurse; CIMS: Care institute of Medical Sciences; CABG: Coronary artery bypass graft; HAIs: Healthcare associated infections; WHO: World Health Organisation.

\section{REFERENCES}

1. Laxminarayan $R$, Duse A, Wattal C, Zaidi AK, Wertheim HF, Sumpradit N, Vlieghe E, Hara GL, Gould IM, Goossens H, Greko C, So AD, Bigdeli M, Tomson G, Woodhouse W, Ombaka E, Peralta AQ, Qamar FN, Mir F, Kariuki S, Bhutta ZA, Coates A, Bergstrom R, Wright GD, Brown ED, Cars O. Antibiotic resistance-the need for global solutions. Lancet Infect Dis. 2013;13(12):1057-98. doi: 10.1016/ S1473-3099(13)70318-9, PMID 24252483.

2. World Health Organization. WHO's first global report on antibiotic resistance reveals serious, worldwide threat to public health.

3. Laxminarayan R, Matsoso P, Pant S, Brower C, Røttingen JA, Klugman K, Davies S. Access to effective antimicrobials: a worldwide challenge. Lancet. 2016;387(10014):168-75. doi: 10.1016/S0140-6736(15)00474-2, PMID 26603918.

4. MunckhofW. Antibiotics for surgical prophylaxis. Aust Prescr. 2005;28(2):38-40. doi: 10.18773/austprescr.2005.030.

5. Misra AK, Gupta R, Bedi JS, Narang M, Garg S, Mail I. Antibiotic prophylaxis for surgical site infection: need of time. Health. 2015;3(3).

6. Australian Commission on Safety and Quality in Health Care. Antimicrobial prescribing practice in Australian hospitals: results of the 2015 National antimicrobial Prescribing Survey. Sydney: ACSQHC; 2016.

7. Ussiri E, Mkony C, Aziz M. Sutured and open clean-contaminated and contaminated laparotomy wounds at Muhimbili National Hospital: a comparison of 
complications. East Cent Afr J Surg. 2004;9(2):89-95.

8. Australian Commission on Safety and Quality in Health Care. Antimicrobial prescribing practice in Australian hospitals: results of the 2015 National antimicrobial Prescribing Survey. Sydney: ACSQHC; 2016.

9. Australian Commission on Safety and Quality in Health Care. National safety and quality health service standards September 2012. Sydney: ACSQHC; 2012.

10. MacDougall C, Polk RE. Antimicrobial stewardship programs in health care systems. Clin Microbiol Rev. 2005 Oct 1;18(4):638-56. doi: 10.1128/CMR.18.4.638656.2005, PMID 16223951.

11. Lee DH, Kim SY, Nam SY, Choi SH, Choi JW, Roh JL. Risk factors of surgical site infection in patients undergoing major oncological surgery for head and neck cancer. Oral Oncol. 2011;47(6):528-31. doi: 10.1016/j.oraloncology.2011.04.002, PMID 21543250

12. Hirakawa H, Hasegawa $Y$, Hanai N, Ozawa T, Hyodo I, Suzuki M. Surgical site infection in clean-contaminated head and neck cancer surgery: risk factors and prognosis. Eur Arch Otorhinolaryngol. 2013;270(3):1115-23. doi: 10.1007/ s00405-012-2128-y, PMID 22865106.

13. Alemkere G. Antibiotic usage in surgical prophylaxis: A prospective observational study in the surgical ward of Nekemte referral hospital. PLOS ONE. 2018 Sep 13;13(9):e0203523. doi: 10.1371/journal.pone.0203523, PMID 30212477.

14. Klinger G, Carmeli I, Feigin E, Freud E, Steinberg R, Levy I. Compliance with surgical antibiotic prophylaxis guidelines in pediatric surgery. Eur J Pediatr Surg.
2015;25(2):199-202. doi: 10.1055/s-0034-1368798, PMID 24683104.

15. Ierano C, Nankervis JM, James R, Rajkhowa A, Peel T, Thursky K. Surgical antimicrobial prophylaxis. Aust Prescr. 2017 Dec;40(6):225-9. doi: 10.18773/ austprescr.2017.073, PMID 29377021.

16. James R. Surgical prophylaxis: data from the Surgical National antimicrobia Prescribing survey 2016. Oral presentation at the National antimicrobial Resistance Forum. Brisbane; Nov 2-3 2017.

17. Bratzler DW, Dellinger EP, Olsen KM, Perl TM, Auwaerter PG, Bolon MK, Fish DN Napolitano LM, Sawyer RG, Slain D, Steinberg JP, Weinstein RA, American Society of Health-System Pharmacists, Infectious Disease Society of America, Surgical Infection Society, Society for Healthcare Epidemiology of America. Clinical practice guidelines for antimicrobial prophylaxis in surgery. Am J Health Syst Pharm. 2013;70(3):195-283. doi: 10.2146/ajhp120568, PMID 23327981.

18. Allegranzi B, Bischoff P, de Jonge S, Kubilay NZ, Zayed B, Gomes SM, Abbas M Atema JJ, Gans S, van Rijen M, Boermeester MA, Egger M, Kluytmans J, Pittet D, Solomkin JS, WHO Guidelines Development Group. New WHO recommendations on preoperative measures for surgical site infection prevention: an evidence-based global perspective. Lancet Infect Dis. 2016;16(12):e276-87. doi: 10.1016/S1473-3099(16)30398-X, PMID 27816413.

19. Harbarth S, Samore MH, Lichtenberg D, Carmeli Y. Prolonged antibiotic prophylaxis after cardiovascular surgery and its effect on surgical site infections and antimicrobial resistance. Circulation. 2000;101(25):2916-21. doi: 10.1161/01. cir.101.25.2916, PMID 10869263.

Article History: Submission Date : 16-06-2021; Revised Date : 06-08-2021; Acceptance Date : 04-09-2021

Cite this article: Khelwade KK, Shrikalp D. A 4-Year Prospective Study on Effect of Antimicrobial Surgical Prophylaxis in the Department of General Surgery. Int. J. Pharm. Investigation. 2021;11(3):319-23. 